\title{
Investigation of the Core-Shell Interface in Gold@Silica Nanoparticles: A Silica Imprinting Approach \\ Saran Poovarodom,${ }^{\dagger}$ John D. Bass, ${ }^{\dagger}$ Son-Jong Hwang, ${ }^{\ddagger}$ and Alexander Katz ${ }^{*}, \dagger$
}

${ }^{\dagger}$ Department of Chemical Engineering

University of California at Berkeley

Berkeley, California 94720-1462

${ }^{\ddagger}$ Division of Chemistry and Chemical Engineering

California Institute of Technology

Pasadena, California 91125

*Corresponding Author

\section{Supplementary Information}

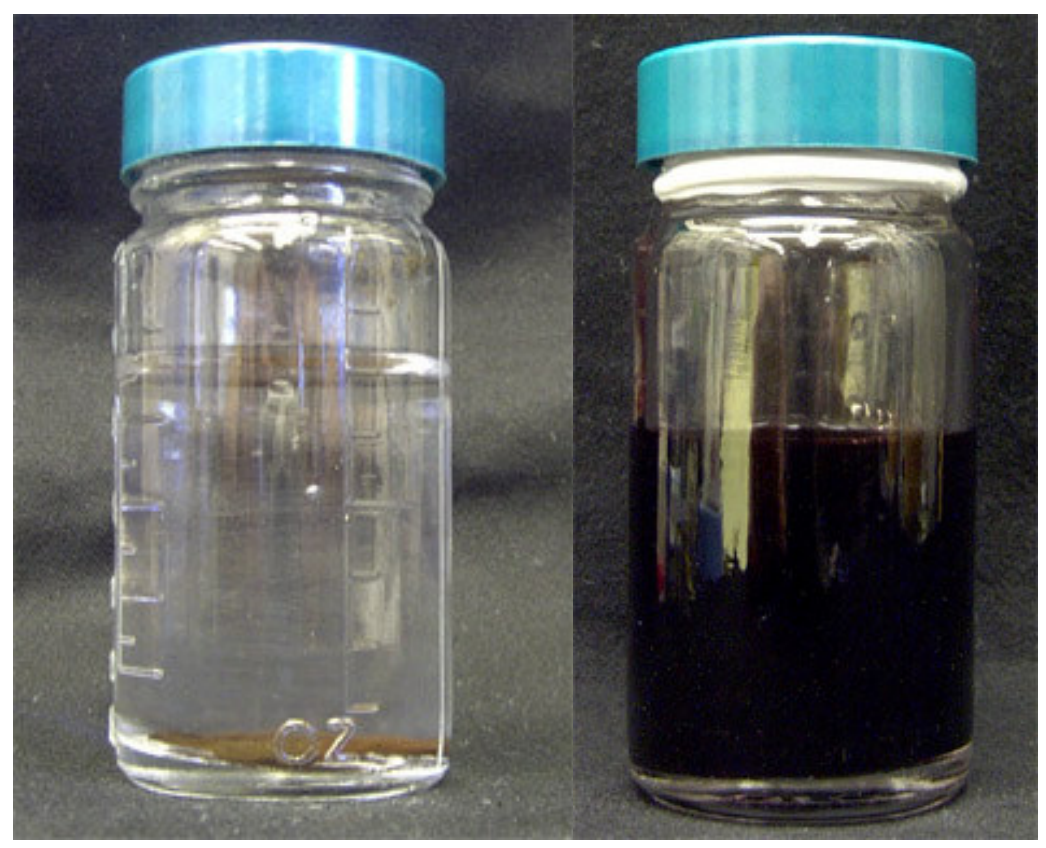

(no amine added)

(with amine added)

Figure 1s. Photographs demonstrating the necessity of APTMS addition for prevention of colloidal gold aggregation during synthesis of gold-silica composite material.

The photograph on the left of Figure 1s represents a procedure that is identical to that described in the manuscript for synthesis of the gold-silica composite material prior to gelation, except that 3-aminopropyl-trimethoxysilane (APTMS) was omitted from gold@silica 
nanoparticle synthesis. Upon concentrating the resulting colloidal gold/silica mixture prior to gelation, colloidal gold aggregation occurs, which is marked by a clear solution with a fine precipitate on the bottom. This is similar to the result obtained by Koboyashi et al. (see Figure 1 of Langmuir 2001, 17, 6375-6379). The photograph on the right of Figure 1s represents the sol resulting from a procedure identical to that described in the manuscript, including the addition of APTMS during synthesis of gold@ silica nanoparticles. The sol retains the characteristic color of isolated $12.5 \mathrm{~nm}$-diameter colloidal gold even after concentration in a rotary evaporator. This can also be verified by the frequency of the surface plasmon resonance absorption band, which is not red-shifted (within $5 \mathrm{~nm}$ ) compared with citrate-stabilized nanoparticles in dilute solution. The photograph on the right in Figure 1s, upon standing less than one week, gelled and was mechanically broken via stirring for synthesizing the particles in Figure 2s.

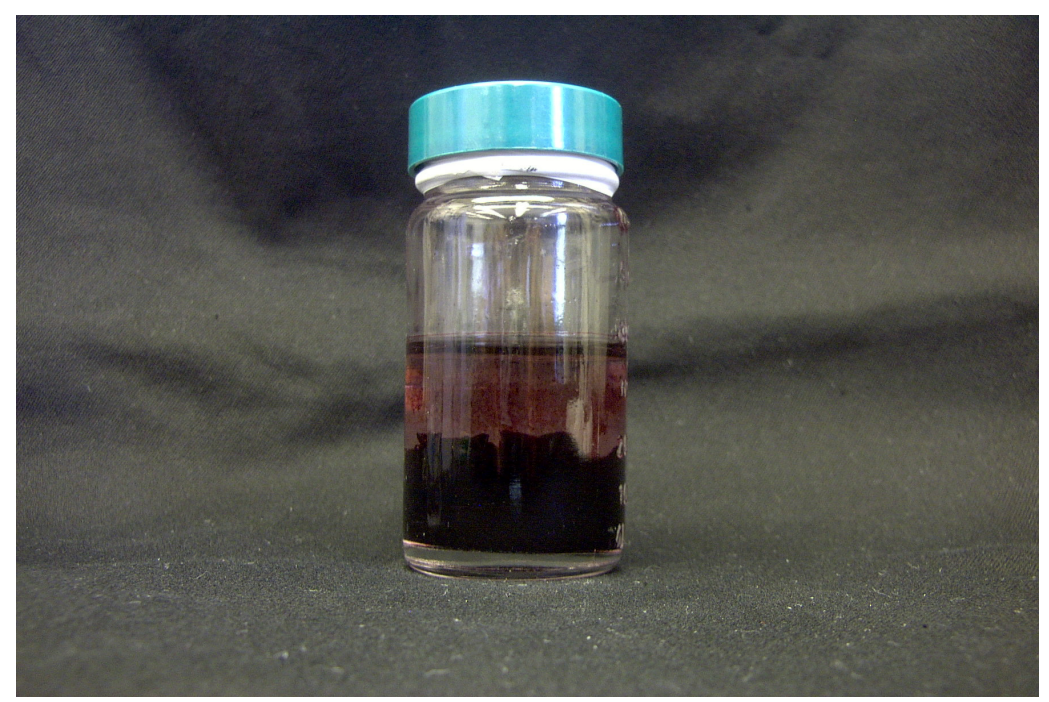

Figure 2s. Photograph demonstrating macroscopic pieces of composite gold-silica gel formed upon letting the solution in the right of Figure 1s stand for less than one week.

The etching process can also be followed via powder X-ray diffraction. This is shown in Figure 3s, which demonstrates the disappearance of peaks associated with gold cores (and 
corresponding to different crystal faces of metallic gold). Note the broad amorphous peak present in the powder pattern of material $\mathbf{1}$ and $\mathbf{2}$, which is characteristic of amorphous silica and no evidence of longer-range order.
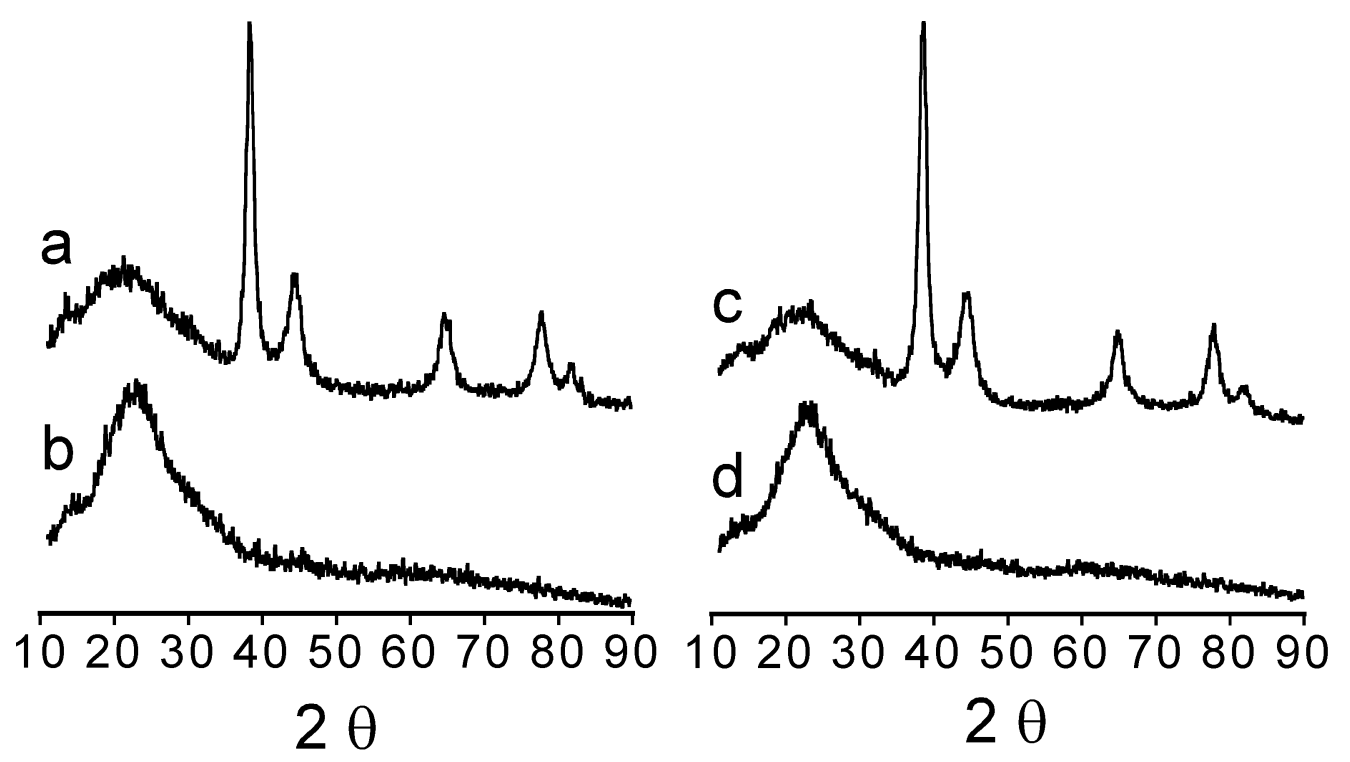

Figure 3s. X-ray diffraction powder patterns of material (a) 1 derived from low $\mathrm{pH}$ silica coating, (b) $\mathbf{2}$ derived from low $\mathrm{pH}$ silica coating, (c) 1 derived from high $\mathrm{pH}$ silica coating, (d) 2 derived from high $\mathrm{pH}$ silica coating.

The dynamics of etching is followed on a single macroscopic gold-silica composite material particle and is shown in Figure 4 of the manuscript. Below in Figure 4s, optical photographs of the etching process on several other gel particles are shown. The length and time scales in the photographs below are similar to those reported for the particle in Figure 4 of the manuscript. 


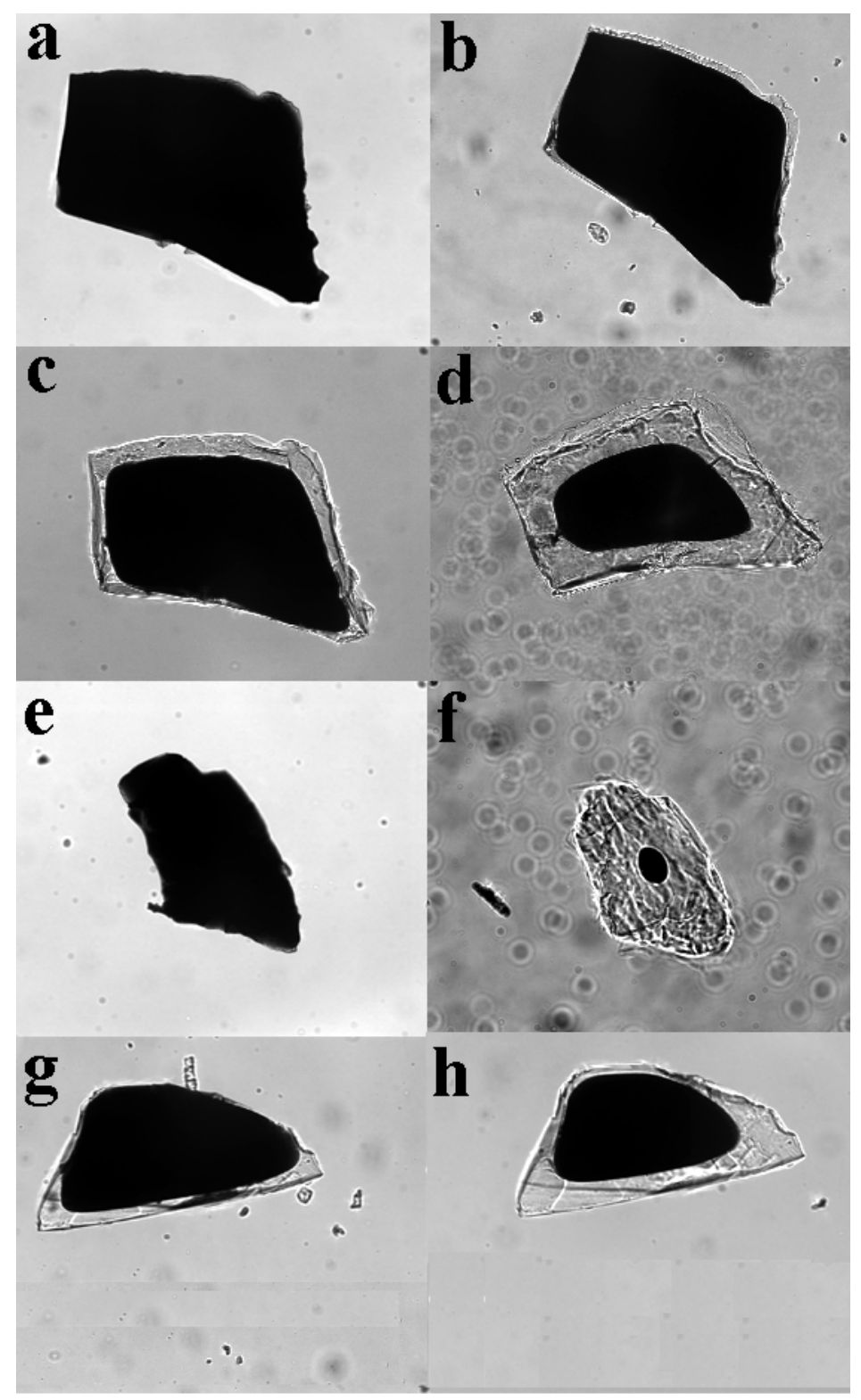

Figure 4s. Etching of macroscopic gold-silica gel particles (each particle is about 100 microns in length). The dynamic course of etching can be followed on the same macroscopic particle of gold-silica composite material in (a-d), (e-f), and (g-h). Note the core-shell morphology of the etching process on these macroscopic particles, which is similar to that shown in Figure 4 of the manuscript.

The gold@silica nanoparticles that were used as building blocks for the synthesis of $\mathbf{1}$ were characterized via TEM as shown in Figure 5s. Upon drying a concentrated solution of the nanoparticles, aggregation of the gold@ silica units ensues, which is quite similar to the type of 
aggregation occurring during the synthesis and, particularly, gelation for synthesizing $\mathbf{1}$. Note the isolated nature of the gold cores in the resulting aggregate shown in Figure $5 \mathrm{~s}(\mathrm{~b}$ and $\mathrm{c})$. This is a direct result of the discrete gold@ silica nanoparticles that were used as building blocks for the synthesis of $\mathbf{1}$.

\section{Calculation of Theoretical Amine Density in 2:}

Synthesis of gold@silica nanoparticles requires $160 \mathrm{~mL}$ of a 0.54 wt \% silica aqueous solution per $20 \mathrm{~mL}$ of a $1 \mathrm{mM}$ APTMS aqueous solution (see Experimental section). Therefore, assuming a quantitative yield of amine incorporation into silica, a mass balance requires:

$0.54 \mathrm{~g}$ silica / $100 \mathrm{~g}$ soln X $160 \mathrm{~mL}$ soln X $1 \mathrm{~g}$ soln / $\mathrm{mL}$ of soln $=0.864 \mathrm{~g}$ silica total per

$20 \times 10^{-3} \mathrm{~L}^{*} 1 \mathrm{mmol}$ aminosilane $/ \mathrm{L}=2 \times 10^{-3} \mathrm{mmol}$ of aminosilane total or, equivalently, $20 \times 10^{-3} \mathrm{mmol}$ aminosilane $/ 0.864 \mathrm{~g}$ silica $=\underline{\mathbf{0 . 0 2 3} \mathbf{~ m m o l} \text { aminosilane } / \mathrm{g} \text { silica }}$

\section{Experimental Conditions for Testing APTMS Anchoring onto Silica Gel from Aqueous Sodium Citrate Solution:}

$400 \mathrm{~mL}$ of an aqueous 2.0 M sodium citrate solution were mixed with $1.2 \mathrm{~g}$ of Selecto brand silica gel (see Chem. Mater. 2002, 14, 3364-3368 for characteristics), to which was finally added $1 \mathrm{~mL}$ of $0.04 \mathrm{M}$ APTMS. Two such solutions were prepared and $\mathrm{pH}$ adjusted to 9.4 (high $\mathrm{pH}$ solution) and 6.4 (low $\mathrm{pH}$ solution). Each of these solutions should result in silica containing a maximum aminosilane loading of $0.03 \mathrm{mmol} / \mathrm{g}$, assuming that all of the aminosilane has covalently bound to the silica surface. Both solutions were shaken for a period of 8 days at room temperature. The silica from each solution was collected via filtration and washed with at least $400 \mathrm{~mL}$ of water, before being dried under vacuum at $105^{\circ} \mathrm{C}$ for a period of 24 hours. Amine site counts were measured by treating a portion of the material with 1.15 equivalents of a 2.2 $\mathrm{mM}$ solution of 5-nitrosalicylaldehyde in acetonitrile, and 5-nitrosalicylaldehyde binding was monitored spectrophotometrically. The results showed $0.03 \mathrm{mmol} / \mathrm{g}$ of nitrosalicylaldehyde attachment on both materials, whereas a control on untreated Selecto silica showed less than $0.005 \mathrm{mmol} / \mathrm{g}$ of binding. 


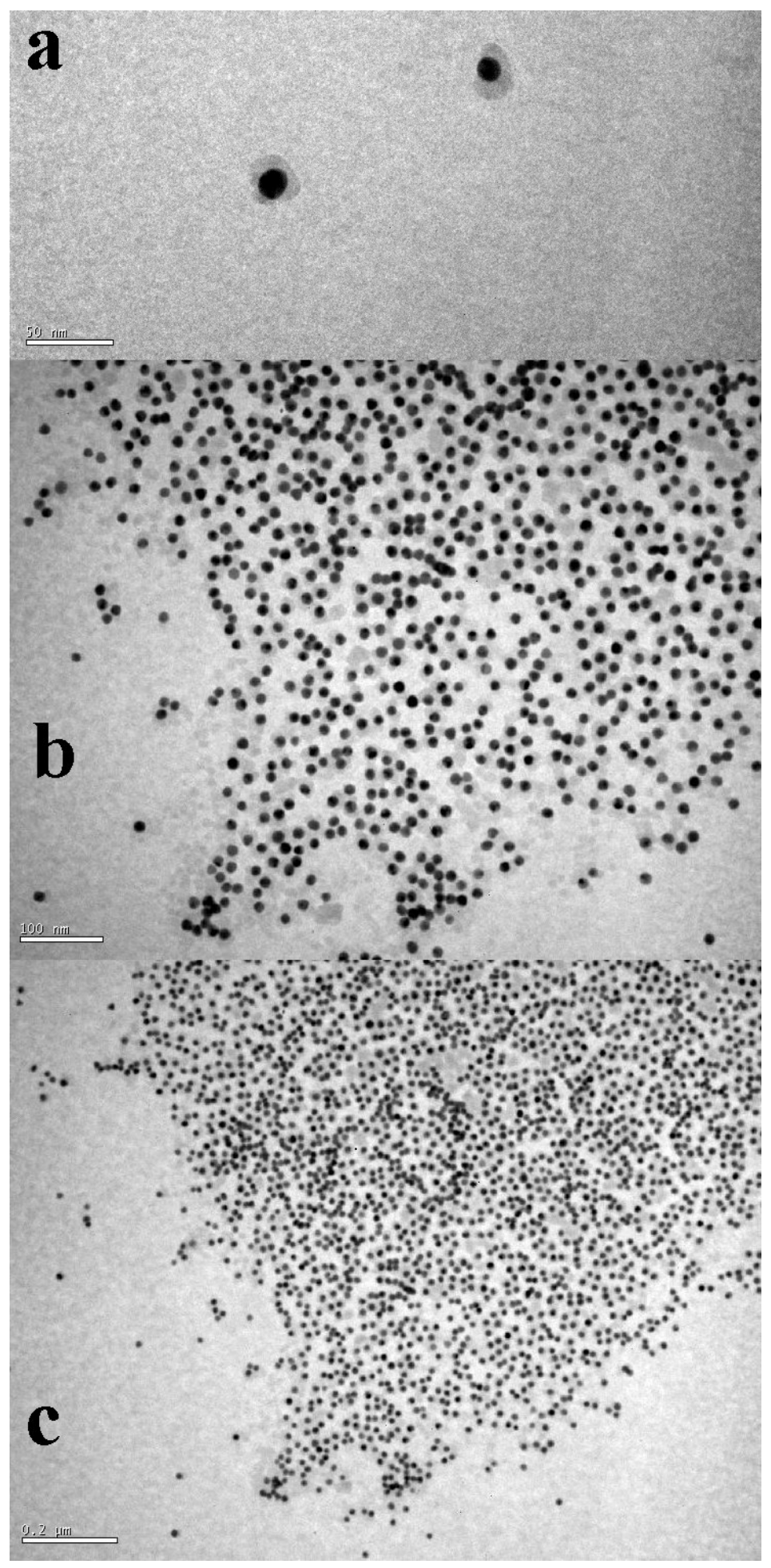

Figure 5s. (a) TEM of isolated gold@silica nanoparticles derived from the high pH silica coating procedure. Upon drying a concentrated solution of these nanoparticles, the aggregate shown in (c) results, of which (b) is a magnified view of the lower left-hand section of (c). Note the discrete nature of the gold cores in aggregates in (b) and (c) that is a direct result of the gold@silica nanoparticle building blocks. 